\title{
ICOSAHEDRAL SYMMETRY AND QUANTUM GRAVITY
}

\author{
J.A.de Wet \\ Box 514,Plettenberg Bay,6600,South Africa
}

Keywords: Jacobi Theta Functions, Riemann Surfaces, Icosahedron, Gravity.

\begin{abstract}
In this note we will show that Theta functions are a solution of the icosahedron equation and also a solution of the Ernst equation for the stationary axisymmetric case of Einstein's gravitational equation.
\end{abstract}

\section{Introduction}

The Theta function was extensively studied in the 19th century by Klein[6] who found a solution for its algebraic background. The icosahedron is the largest Platonic solid with 20 triangular faces and 12 vertices. It can be rotated into itself about 6 diagonals described by the Icosahedral group which has the Exceptional Lie algebra E8 by the McKay correspondence. The subalgebra E6 has recently been employed by de Wet [5] as a basis for the Standard Model of the elementary particles and can be described by Theta functions[2]. Furthermore representations of E8 have also been used to cover supersymmetry [4].

Having introduced E8 as a home for the elementary and supersymmetric particles we will now go on to study the Jacobi Theta functions that yield the number of vectors or vertices in shells of the toroidal graphs of E6 and E8 as well as providing a solution of the Ernst equation in the case of E8 found by Korotkin [8,9].

\section{Theta Function Solutions}

On page 2596 Korotkin [7] considers the simplest elliptic solution of the Ernst equation for 4 double-valued sheets on a toroidal Riemann surface determined by 4 branch points which is precisely the graph of the 8 shells of E8 with 240 vertices to be described below. Specifically the branch points are

$$
(\lambda-\xi)(\lambda-\xi)(\lambda-E)(\lambda-E)-
$$

where $\xi=(z+i \rho)$ is a factor of automorphy and $E$ is independent of $E, K=(\xi, \xi)$ is a vector of Riemann constants.

Specifically Klein,Korotkin and Shramchenko [8] employ the following solution of the Ernst equation

$$
\theta_{p q}=\sum_{m=2 \theta} \exp [i \pi(B((p+m),(p+m))+2 i \pi((p+m),(q+z)]
$$

where $\mathrm{g}=1$ for the $\mathrm{E}_{8}$ torus in our case and we are looking at a line-bundle on $\mathrm{C}^{1}$ where $\mathrm{p}, \mathrm{q}$ are vectors on the Riemann surface and B is the matrix of their cycles.

Then according to Conway and Sloane [2] the Jacobi Theta function for $\mathrm{E}_{8}$ with 240 vertices is

$$
\theta=\sum_{m=1} N_{m} q^{m}=1+240 q^{2}+2160 q^{4}+\ldots \text { with } \mathrm{q}=\exp (\pi \mathrm{iz})
$$

and a periodic function converging rapidly. Here $\xi=\mathrm{Nm}$ is a factor of auto- morphy of rank 1 which in the case of a lattice of a Lie group $E_{8}$ is a line bundle or vector space $B((p+m),(p+m))$ of (2) specified by the scalars of 
(3) which yield the number of vectors or vertices $\mathrm{N}_{m}$ in the sucessive shells of around the origin of $\mathrm{E}_{8}$ which is 240 when $\mathrm{m}=2$ (see [3] Table 4.8)

In Table 4.9 Conway and Sloane list the number of vertices in successive shells in the lattice around the central hole for $\mathrm{E}_{6}, \mathrm{E}_{7}, \mathrm{E}_{8}$ with norm $\mathrm{m}$. In the case of $\mathrm{E}_{8}$ and $\mathrm{m}=2$ there are 240,made up of 4 shells of 2 orbits that can change sign, Turning to Table 2.10, 224 vertices are of the form $( \pm 1, \pm 1,0,0,0,0,0,0)$ where are 7 ways of choosing \pm 1 among 7 places if we fix one \pm 1 which can occupy 8 places, making a total of 56 choices. But since we also have the 4 possibilities $(+1,+1)(+1,-1)(-1,+1)(-1,-1)$ and their permutations with 6 zeros there are altogether $4 \times 56=224$ possibilities. However Conway and Sloane ([2], p 121) associate this lattice with enclosing a deep hole surrounded by another 6 vertices to provide the 240 vertices.

\section{Conclusion}

The lattice of $E_{8}$ is a torus or loop so is the same space employed by Sundance Bilson- Thompson and Smolin to describe Loop Quantum Gravity [1] which illustrates the fundamental importance of Icosahedral symmetry. Specificaly the Icosahedron is a Platonic solid illustrating the reality of mathematical concepts originally considered as Forms by Plato(cf.Penrose [9] ch,3)

\section{References}

[1] Sundance O. Bilson-Thompson,Fotini Markopoulo and Lee Smolin, Quantum Gravity and the Standard Model,online,June(2014).

[2] J.H.Conway and N.J.A,Sloane, Sphere Packings,Lattices and Groups, Third Ed.,Springer(1993)

[3] H.S.M.Coxeter,Regular Complex Polytopes,Camb.Univ.Press,Second Ed.(1991)

[4] J.A. de Wet, Icosahedral Supersymmetry, online, Published by Int.Soc. of Frontier Science,Oct.(2014).

[5] J. A. De Wet, A Standard Model Algebra, Int. Mathematical Forum,v8, (2013) 111-1117, HIKARI Ltd. http://dx.doi.org/10.12988/imf.2013.3477.

[6] Felix Klein, Lectures on the Icosahedron,Dover(2003).

[7] D.A.Korotkin, Elliptic solutions of stationary axisymmetric Einstein equation, Class.Quantum Grav.10(1993)2587-2613.

[8] C.Klein,D.Korotkin and V.Shramchenko, Ernst equation, Fay identities and variatinal formulas on hyperelliptic curves, arxiv: math- phys/0401055v1(2008).

[9] Roger Penrose, The Emperor's New Mind.Oxford Univ.press (1989). 